\title{
A tripartite virulence complex in Toxoplasma
}

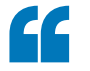

these kinases

function

synergistically

to block IRG

accumulation

on the PVM
Toxoplasma gondii is a common intracellular parasite that infects a wide range of mammalian hosts. Following invasion, host interferon- $\gamma$ $($ IFN $\gamma)$-induced immunity-related GTPases (IRGs) accumulate on the membrane of the parasitophorous vacuole that surrounds the parasite in host cells, which leads to vacuole disruption and parasite death. In mice, T. gondii evades this immune response by recruiting a rhoptrysecreted kinase, ROP18, to the parasitophorous vacuole membrane (PVM) to phosphorylate host IRGs and thus block their accumulation. The pseudokinase ROP5 also interacts with IRGs and enhances the kinase activity of ROP18 and thus has a crucial role in virulence. Etheridge et al. now identify the previously uncharacterized kinase ROP17 as a novel component of ROP5-

containing complexes and show that ROP5,

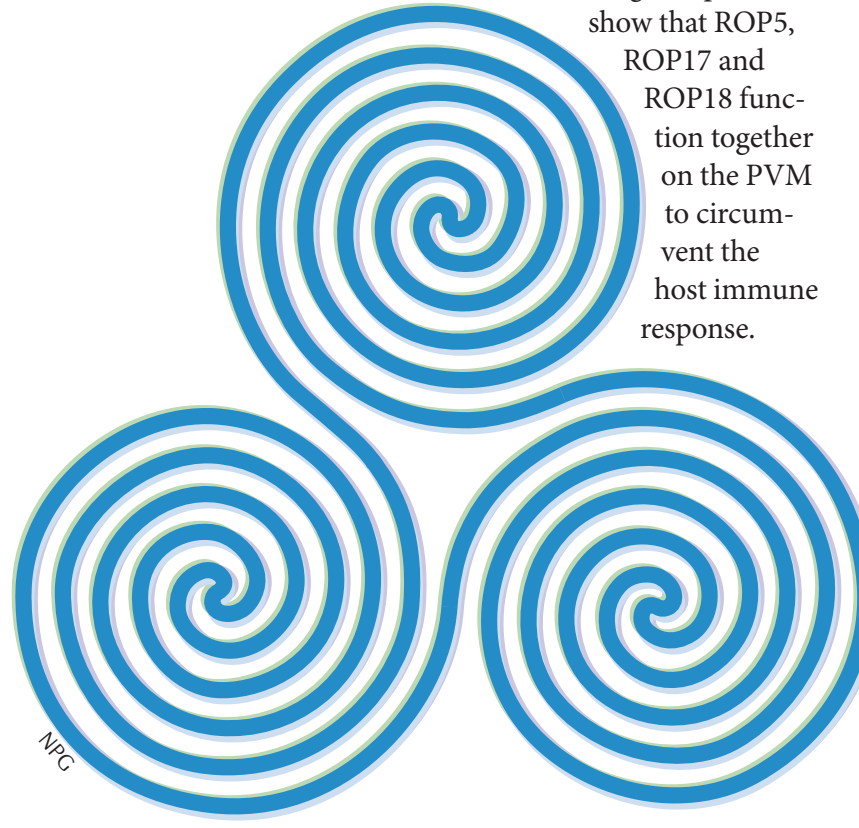

Previous results showed that, although deletion of ROP18 in virulent type I T. gondii strains had only a modest effect on virulence, deletion of ROP5 resulted in avirulent mutants, which suggests that ROP5 regulates additional virulence effectors. To identify novel ROP5-binding factors, the authors used tandem affinity purification (TAP) tagging and found that ROP5 exists in a heterogeneous complex that contains not only ROP18, as expected, but also ROP17, which is a previously uncharacterized protein. Fluorescence microscopy studies in infected human host cells revealed that ROP5 and ROP17 colocalize on the PVM, which suggests that there is a functional link between these two proteins.

Like ROP18, ROP17 contains a catalytic triad and is thus predicted to be a serine/threonine kinase. Consistent with this, the authors showed that native ROP17-containing complexes had catalytic activity in vitro. In addition, the kinase activity of the ROP17 complex was greater than that of recombinant ROP17, which suggests that additional factors contribute to ROP17 activity. However, unlike ROP18, the catalytic activity of ROP17 was not directly dependent on ROP5 in vitro.

Next, to examine the contribution of ROP17 to virulence, the authors generated a series of deletion mutants; deletion of either ROP17 or ROP18 had only a modest effect on virulence in inoculated mice, whereas the virulence of a mutant that lacked both ROP17 and ROP18 was more severely attenuated.
Importantly, mice in which the IFN $\gamma$ signalling pathway or the IRG pathway was disrupted were susceptible to challenge with the single or double mutants. This led the authors to propose that ROP17 and ROP18 target a common host pathway. In agreement with this, ROP17, like ROP18, was shown to phosphorylate the conserved switch region I (SWI) in the GTPase domain of IRGs. However, ROP17 and ROP18 were found to target distinct threonine residues in the SWI region of these host proteins, which suggests that these kinases function synergistically to block IRG accumulation on the PVM. Indeed, parasites that were deficient in both ROP17 and ROP18 were more susceptible to clearance by IFN $\gamma$-activated macrophages than single deletion mutants. Finally, the authors showed that ROP17 preferentially phosphorylates IRG oligomers, which leads to their disassembly into monomers and dimers and thus may contribute to the protection of the parasitophorous vacuole in infected cells.

This study reveals a model whereby the virulence effectors ROP5, ROP17 and ROP18 function synergistically to target host IRGs, which promotes T. gondii evasion of the mouse immune response.

Andrea Du Toit

ORIGINAL RESEARCH PAPER Etheridge, R. D. et al. The Toxoplasma pseudokinase ROP5 forms complexes with ROP18 and ROP17 kinases that synergize to control acute virulence in mice. Cell Host Microbe http://dx.doi.org/10.1016/j. chom.2014.04.002 (2014)

FURTHER READING Hunter, C. A. \& Sibley, L. D.

Modulation of innate immunity by Toxoplasma gondii virulence effectors. Nature Rev. Microbiol. 10, 766-778 (2012) 